with information about health benefits and selecting personally relevant motives and social support sources regarding physical activity, 3) an action planning intervention in which participants specified which physical activities they would do, when, where and for how long and 4) a combination of these interventions. Prior to the intervention, participants' leisure time physical activity in the past week was measured with items from the SQUASH. Also behavioural intentions, expected social support and planning behaviour with regard to leisure time physical activity were measured. Directly after the intervention, positive outcome expectations and self-efficacy were measured. After a week, physical activity measures were administered again.

Results: 208 Participants $(71 \%$ female; mean age 62 years; $40 \%$ with a rheumatic disease) completed the study. Figure 1 displays the average change in physical activity for each intervention. Multiple regression analyses showed that both the motivational intervention $(\beta=0.22)$ and action planning $(\beta=0.31)$ significantly predicted a positive physical activity change and that these effects appeared additive as opposed to catalytic. The effects were not moderated by intention, expected social support or planning behaviour, nor mediated by outcome expectations or self-efficacy. The motivational intervention's effectiveness was weakly correlated with the number of personally relevant exercise motives identified $(r=0.22)$ and the action planning's effectiveness was weakly related to the number of plans made $(r=0.19)$ and to one's outcome expectations after the intervention $(r=0.21)$.

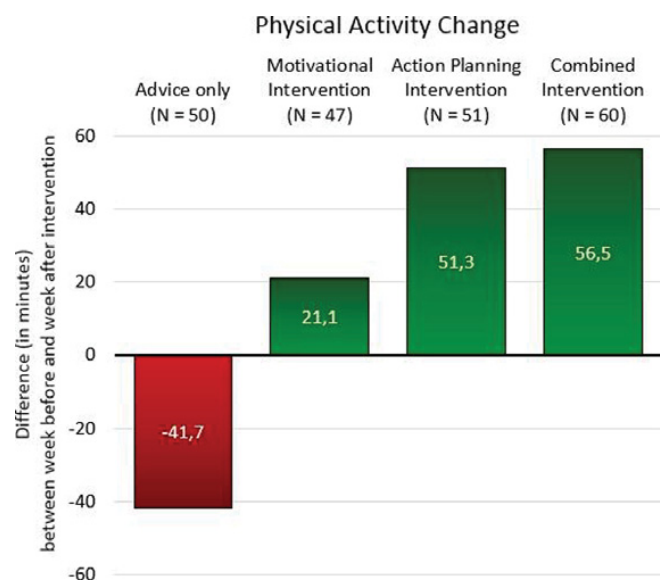

Conclusions: These data suggest that using a motivational or action planning intervention seems beneficial for increasing physical activity in patients with a chronic disease, independent of one's intentions, expected social support and planning behaviour. The data also suggest that action planning is preferable over a motivational intervention and that the added value of a motivational intervention is minimal if action planning is used.

This study was limited by only measuring physical activity after a week and by using self-report. Future studies should investigate longer term effects, other potential moderators and mediators and interaction effects between other promising behavioural change methods.

Disclosure of Interest: None declared

DOI: 10.1136/annrheumdis-2017-eular.2137

\section{THU0750-HPR THE EFFECTIVENESS OF THERAPEUTIC FOOTWEAR IN PATIENTS WITH RHEUMATOID ARTHRITIS: A SYSTEMATIC REVIEW AND META-ANALYSIS}

M. Tenten-Diepenmaat ${ }^{1}$, M. Leeden van $\operatorname{der}^{1,2}$, T. Vliet Vlieland $^{3}$, L.D. Roorda ${ }^{1}$, J. Dekker ${ }^{2,4} .{ }^{1}$ Amsterdam Rehabilitation Research Center, Reade; ${ }^{2}$ Department of Rehabilitation Medicine, VU University Medical Center, Amsterdam;

${ }^{3}$ Department of Orthopaedics, Rehabilitation and Physical Therapy, Leiden

University Medical Center, Leiden; ${ }^{4}$ Department of Epidemiology and

Biostatistics, Amsterdam Public Health research institute, VU University Medical Center, Amsterdam, Netherlands

Background: Therapeutic footwear is recommended in guidelines for the treatment of foot problems in patients with rheumatoid arthritis (RA) and commonly prescribed and frequently used, especially in patients with established RA, foot deformities or erosions in foot joints.

Objectives: The objectives of this study were to summarize the effectiveness of therapeutic footwear on foot function, pain, physical functioning, health-related quality of life, adherence, adverse events and patient satisfaction in patients with RA.

Methods: All randomized controlled trials, randomized controlled cross-over trials, (quasi-experimental) clinical trials, and uncontrolled studies investigating the effect of therapeutic footwear in patients with RA related foot problems were included. Therapeutic footwear consists of custom-made or ready-made shoes. A literature search was conducted in The Cochrane Central Registry for Controlled Trials (CENTRAL), PubMed, EMBASE and PEDro up to January 19, 2017. Selection and inclusion of articles, data extraction (using a standardized template) and assessment of methodological quality (using a checklist for between-group and within-group comparisons) was conducted by two independent reviewers. Quantitative data analyses was conducted, when quantitative data analysis was not possible qualitative data analysis was performed.

Results: Thirteen studies were identified. In five studies, one of which was of high quality, between-group differences were reported. In twelve studies, of which two were of high quality, within-group differences were reported. Qualitative datasyntheses for the within-group differences of custom-made therapeutic footwear resulted in weak evidence for the reduction of foot pain and improvement of physical functioning. Quantitative data-analyses of the within-group differences of ready-made therapeutic footwear resulted in a medium to large effect for the reduction of foot pain (SMD $-0.68,95 \% \mathrm{Cl}-1.00$ to $-0.37 ; \mathrm{P}=0.0001 ; 162$ participants), and a small to medium effect for the improvement of physical functioning (SMD $-0.32,95 \% \mathrm{Cl}-0.62$ to $-0.02 ; \mathrm{P}=0.04 ; 128$ participants). Qualitative data-analyses of the between-group differences of ready-made therapeutic footwear resulted in inconclusive evidence for foot pain and physical functioning.

Conclusions: In within-group designs, there is weak evidence for the reduction of foot pain and improvement of physical functioning after wearing custom-made therapeutic footwear. Furthermore, in within-group designs there is a medium to large effect of ready-made therapeutic footwear on the reduction of foot pain, and a small to medium effect on the improvement of physical function. Controlled, between-group designs resulted in inconclusive evidence. A definite RCT is necessary to investigate the between-group effectiveness of therapeutic footwear in patients with RA.

Disclosure of Interest: None declared

DOI: 10.1136/annrheumdis-2017-eular.5809

\section{THU0751-HPR EFFECTIVENESS OF FOOT ORTHOSIS IN PATIENTS WITH RHEUMATOID ARTHRITIS RELATED TO QUALITY OF LIFE AND PAIN. A SYSTEMATIC REVIEW AND META-ANALYSIS}

G. Gijon-Nogueron, L. Ramos-Petersen, S. Garcia-Mayor, J.M. Morales-Asencio. Nursing and Podiatry, University of Malaga, Malaga, Spain

Background: Foot pain and deformity is almost ubiquitous in RA and results in considerable physical and psychosocial impairment [1]. Epidemiological studies consistently suggest a $90 \%$ prevalence of foot pain despite advances in pharmacological therapy [2]. Mechanical and other non-pharmacological interventions such as orthoses and footwear, have an important role in managing foot pathology in patients with their systemic disease controlled $[1,3]$. The effectiveness of treatment with insoles, especially in early periods, was studied in a randomized controlled trial, which results suggested an immediate clinical improvement, reducing foot pain, disability and limited functionality.[1]

Objectives: The aim of this study is the effectiveness of foot orthosis in patients with rheumatoid arthritis in terms of quality of life and pain.

Methods: A systematic review and meta-analysis was conducted of randomized controlled trials.

Participants: Patients with rheumatoid arthritis were included. The criteria of exclusion were Juvenile Rheumatoid Arthritis, analysis of gait,

Intervention: Studies had to compare foot orthosis

Comparison: Other type of treatments, other type of foot orthosis, sham

Outcomes: Evaluation of Pain or Quality of life with any tool that measure this outcomes

The search was conducted in Cochrane, CINAHL, PubMed, EMBASE, LILACS, and Cuiden. An independent peer review was carried out. The Mesh term and fields used were foot, ankle, joint, rheumatoid arthritis, foot, orthosis, insole, foot orthosis.

Results: After the analysis of 71 studies, 4 were included for the systematic review. The 4 studies enrolled 285 participants. Follow-up periods varied from 6 to 30 months.

Only two studies were included in the meta-analysis [4,5], both of them with pain (measured with Foot Function Index) as the selected outcome.

A meta-analysis of the two trials showed that use of FO resulted in a nonsignificant improvement in disability compared with control (MD $(95 \% \mathrm{Cl}): 4.37$ $(-6.24,14.98) ; \mathrm{N}=64)$ (Figure 1).

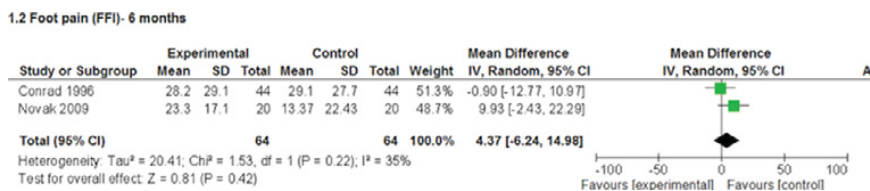

Conclusions: Foot orthoses showed improvements in pain and disability/quality of life, but no significant differences between groups were found.Future research needs to increase the number of RCTs in this topic because results are not conclusive.

\section{References:}

[1] Woodburn J, Barker S, Helliwell PS. A randomized controlled trial of foot orthoses in rheumatoid arthritis. J Rheumatol. 2002;29:1377-83.

[2] Otter SJ, Lucas K, Springett K, Moore A, Davies K, Young A, et al. Comparison 